Código JEL: M4

\title{
Impacto de la Aplicación de la NIC 40 en los Estados Financieros de México
}

\author{
Josernesto GÓMEZ CASTILLO \\ Universidad Complutense de Madrid \\ josernesto@hotmail.com \\ Mercedes RODRÍGUEZ PAREDES \\ Universidad Complutense de Madrid \\ paredes@ccee.ucm.es
}

Recibido: 13-07-2015

Aceptado: 22-10-2015

\section{RESUMEN}

La imagen fiel de los estados financieros es una de las características más importantes que debe emanar de la información financiera, es por ello que una empresa debe optar por la normatividad que refleje esta situación de la mejor manera. En este contexto, el método de medición que se elija para determinar el valor de los elementos de los estados financieros es muy importante, es por ello que el presente estudio tiene como objetivo básico analizar el impacto financiero que tiene el uso del modelo del valor razonable establecido en la NIC 40 para la valoración de las propiedades de inversión planteando para ello el caso de una empresa mexicana que aplica las normas de información financieras nacionales las cuales no le permiten el uso del citado valor razonable. La metodología que se utiliza se centra en el análisis y resultados de las siguientes áreas de trabajo: i) la emisión de estados financieros de acuerdo a las normas de información financiera nacional e internacional; ii) el impacto en el activo no corriente y en el beneficio y iii) el impacto impositivo. 
Finalmente se analiza en forma general la implicación positiva del uso del valor razonable dentro de la empresa en estudio.

Palabras clave: Valor Razonable, Norma Internacional de Contabilidad 40, Norma de Información Financiera B-6, Activo No Corriente y Beneficio.

\title{
Impact of Application of IAS 40 in the Financial Statements of Mexico
}

\begin{abstract}
Faithful representation of financial statements is one of the most important characteristics of financial information. This is why an enterprise must adopt a normativity which adequately this condition. In the ligth of this context, the measurement method to be selected in order to determine the value of financial states is utmost crucial. Thus the present study aims at analyzing the financial impact of using the reasonable value model, established by IAS 40, as a means to value investment properties within a mexican enterprise which applies the financial information national standard which do not allow the use of the aformentioned reasonable value. The analysis of this study focuses on i) issuance of financial statements according to national and international standards ; ii) the impact on the non current assets and earnings and iii) the mandatory impact. Finally, an anlysis of the positive implications of the use of a razonable value within the enterprise being reasearched.
\end{abstract}

Keywords: Fair Value, International Financial Reporting Standards 40, Financial Reporting Standard B-6, Non-current assets, Earning.

Sumario: 1. Introducción 2. Normatividad Financiera Internacional 3. Normatividad Financiera Mexicana 4. Diferencias entre la NIC 40 y la NIF 65. Impacto del Uso de la NIC 40 en los Estados Financieros 6. Impacto Impositivo del uso de la NIC 40 7. Conclusiones.

\section{INTRODUCCIÓN}

Las inversiones inmobiliarias en terrenos y edificios son una importante forma de inversión, que permite no solo conservar el valor del dinero invertido sino incrementar el valor del mismo con el paso del tiempo por medio de la plusvalía. En este sentido, según información de la Sociedad Hipotecaria Federal, se indica que el valor de la vivienda en la Ciudad de México (DF) en el 2014 aumentó un $8.02 \%$ con respecto al 2013 , porcentaje que se sitúa arriba de la inflación registrada para el año 2014 en 3.94\%.

Es por ello que la inversión en inmuebles se proyecta como una gran oportunidad para la obtención de beneficios económicos que aumenten las 
utilidades de las entidades en especial en una economía como la mexicana, que en 2015 ha sufrido una fuerte depreciación del peso ante el dólar, pasando de $\$ 14.73$ pesos por dólar en diciembre de 2014 a $\$ 16.91$ pesos por dólar en agosto de 2015, lo que representa una pérdida de valor del peso del $15 \%$ y donde la inversión en instrumentos financieros representa una fuerte volatilidad por la caída del precio de petróleo y en general de la economía mexicana que ha disminuido su proyección de crecimiento para 2015 de un $3 \%$ a $4 \%$ a un $2.5 \%$ y $3.5 \%$ según datos del Banco de México (Banxico).

Ante esta situación es indispensable que los estados financieros de una entidad registren todos los eventos económicos de la manera más fiable posible, es por ello que el presente trabajo tiene la finalidad de demostrar el impacto financiero que sufre una entidad mexicana al aplicar las Normas Internacionales de Información Financiera (IFRS siglas en inglés) en lugar de las Normas de Información Financieras Mexicanas (NIF) en lo relativo a inversiones inmobiliarias.

Para determinar el citado impacto financiero, se parte del análisis de la NIC 40 (Propiedades de inversión), siguiendo con el estudio de las NIF mexicanas en lo relativo a las inversiones inmobiliarias, para determinar las diferencias de ambas normatividades respecto a los inmuebles. Para ello, se presentan tanto el Estado de Situación Financiera como el Estado de Resultado Integral con base a IFRS y a NIF mexicanas para que, por medio del análisis económico financiero se determine cuál de las dos normatividades representa un beneficio mayor para las entidades mexicanas.

De igual forma se presenta también un análisis fiscal del impacto de aplicar IFRS o NIF mexicanas a las inversiones inmobiliarias, para finalizar con una serie de conclusiones.

\section{NORMATIVIDAD FINANCIERA INTERNACIONAL}

\section{Aspectos generales}

La norma internacional de contabilidad NIC 40 (Propiedades de Inversión) tiene el objetivo de establecer el tratamiento contable de las inversiones inmobiliarias. El termino de propiedad de inversión es definido por la NIC 40 como aquellas "propiedades (terrenos o edificios, considerados en su totalidad o en parte o ambos) que se tienen (por parte del dueño o por parte del arrendatario que haya acordado un arrendamiento financiero) para obtener rentas, apreciación del capital o ambas" (IFRS, 2014), en lugar de tenerlas para los siguientes fines:

1.- Uso en la producción

2.- Suministro de bienes

3.- Fines administrativos; o

4.- De venta

En consecuencia, el uso de la propiedad determinará su clasificación dentro del Estado de Situación Financiera, y se clasificarán como propiedades de inversión, 
aquellas inversiones que estén destinadas a generar rentas o plusvalía, entre otros casos tenemos los siguientes (la clasificación que se presenta está en relación con el uso que tenga el inmovilizado en sí):

\section{Tabla 1}

Inversiones Inmobiliarias clasificadas como "Propiedades de Inversión"

1.- Terreno adquirido para obtener plusvalía a largo plazo y no para venderse

2.- Terreno adquirido para un uso futuro no determinado

3.- Edificio propiedad de la entidad que está arrendado

4.- Edificio desocupado que se tiene para ser arrendado

5.- Inmuebles que están en proceso de construcción o mejora con la finalidad de arrendarlos

Las propiedades que sean adquiridas para un uso distinto al de generar rentas o plusvalía y tengan una finalidad específica dentro de las actividades de las entidades ya sea en el proceso productivo o en la prestación de servicios, deberán clasificarse como propiedad, planta y equipo, en cuyo caso, se aplicará la NIC16 o cuando sean adquiridos con el propósito de venta como inventarios, que tendrá que aplicarse la NIC2, y por lo tanto, claramente, se establece que la aplicación de la normativa es diferente. Los siguientes casos son ejemplos de lo mencionado: (IFRS, 2014)

\section{Tabla 2}

Inversiones Inmobiliarias clasificadas como "Propiedad, planta y equipo (NIC16) ó Inventarios (NIC2)"

\begin{tabular}{|l|}
\hline $\begin{array}{l}\text { 1.-Propiedades destinadas para suministro de mercancías, servicios o fines administrativos } \\
\text { relacionados con la actividad de la empresa (NIC16) }\end{array}$ \\
\hline 2.-Propiedades que están siendo construidas o desarrolladas por terceros (NIC11) \\
\hline 3.-Propiedad ocupada por el propietario (NIC16) \\
\hline 4.-Propiedades arrendadas a otra entidad en régimen de arrendamiento financiero (NIC16) \\
\hline 5.-Propiedades en proceso de construcción o desarrollo con fines de venta (NIC2) \\
\hline
\end{tabular}

\section{Doble uso}

Existen casos especiales en los que, el inmueble tiene un doble uso, por una parte sirve para generar rentas y plusvalía y por otra parte tiene fines administrativos, productivos, de venta o de servicios, en estos casos la norma establece que se debe proceder de la siguiente forma:

1.- Si las partes pueden venderse por separado, se contabilizará la parte que se utilice para generar rentas o plusvalía como propiedad de inversión y la parte que sea utilizada con fines administrativos, productivos, etc., se clasificará como propiedad, planta y equipo (IFRS, 2014). 
2.- Si las partes no pueden separarse, se contabilizará como propiedad de inversión únicamente si aquella parte utilizada con fines distintos al de generar renta o plusvalía representa una porción insignificante de la totalidad del inmueble, en caso contrario se tendrá que contabilizar la totalidad del inmueble como propiedad, planta y equipo (IFRS, 2014).

En este caso, es fundamental desde el punto de vista de la gestión empresarial que se pueda realizar una separación en función del uso que se le de al determinado activo (Asesor empresarial, 2014, 4)

\section{Tabla 3}

Ejemplos de Propiedades Con Doble Uso

\begin{tabular}{|l|c|c|}
\hline Propiedad & $\begin{array}{c}\text { Partes que pueden } \\
\text { clasificarse como inversiones } \\
\text { inmobiliarias }\end{array}$ & $\begin{array}{c}\text { Partes que no pueden } \\
\text { clasificarse como } \\
\text { inversiones inmobiliarias }\end{array}$ \\
\hline $\begin{array}{l}\text { Complejo } \\
\text { Hotelero }\end{array}$ & $\begin{array}{c}\text { Tiendas minoristas separadas; } \\
\text { Bloque de oficinas }\end{array}$ & $\begin{array}{c}\text { Habitaciones de hotel } \\
\text { Instalación de restaurante }\end{array}$ \\
\hline Aeropuertos & $\begin{array}{c}\text { Edificios separados como } \\
\text { hoteles, oficinas, etc. }\end{array}$ & $\begin{array}{c}\text { Concesiones minoristas en la } \\
\text { terminal del aeropuerto }\end{array}$ \\
\hline
\end{tabular}

Fuente: KPMG International Financial Reporting Group. Las NIIF comentadas (2012).

\section{Servicios complementarios}

Un caso similar ocurre con la prestación de servicios complementarios a los ocupantes de un inmueble, si estos servicios son un componente insignificante del contrato como en el caso de servicios de mantenimiento y vigilancia, se seguirá contabilizando el inmueble con base a la NIC 40, en caso de que los servicios prestados representen un componente altamente significante del contrato como sería en la gestión de un hotel, el inmueble se tendrá que contabilizar como propiedad, planta y equipo y por lo tanto, la norma a aplicar sería la NIC16..

\section{Reconocimiento inicial}

Al igual que otros activos las propiedades de inversión se reconocerán cuando: 1.- Sea probable que los beneficios económicos futuros que estén asociados con tales propiedades fluyan hacia la entidad; $y$

2.- El costo de dicha propiedad de inversión pueda ser medido en forma fiable (IFRS, 2014).

Según este criterio de reconocimiento, la entidad evaluará todos los costos de sus propiedades de inversión en el momento en que incurran en ellos. Estos costos comprenderán tanto aquellos en que ha incurrido inicialmente así como aquellos en los que incurre posteriormente relativo a la adquisición o construcción de las propiedades, dentro de los citados costos quedan excluidos los de mantenimiento diario de la propiedad. 
La medición inicial de las propiedades de inversión será al costo, el cual estará integrado por los siguientes elementos:

1.- Precio de compra o construcción.

2.- Honorarios profesionales por servicios legales.

3.- Impuesto por traspaso de las propiedades; y

4.- Otros costos asociados a la transacción.

Los desembolsos posteriores a su reconocimiento solo se agregarán al valor de la propiedad si éstos crean la posibilidad de obtener un beneficio económico mayor al que se obtiene actualmente y, en caso contrario se clasificarán como gasto. Una propiedad de inversión no verá incrementado su costo por los gastos de puesta en marcha, pérdidas de operación incurridas antes de que se logre el nivel planeado de ocupación así como las cantidades anormales de desperdicio, mano de obra u otros recursos en la construcción o desarrollo de la propiedad (IFRS, 2014).

\section{Reconocimiento posterior}

Con posterioridad al reconocimiento inicial, la entidad deberá elegir una política contable uniforme, con el fin de valorar las propiedades de inversión utilizando uno de los dos modelos siguientes (Ramírez Orellana, A, 2007):

A) Modelo de Valor Razonable; o

B) Modelo del Costo

\section{Modelo de valor razonable}

La norma señala que las entidades deberán preferentemente escoger el Modelo de Valor Razonable como política contable de valoración, excepto para aquellas inversiones inmobiliarias que desde el momento de su reconocimiento inicial presenten evidencias que su valor razonable no puede determinarse con fiabilidad, ya sea porque las transacciones de mercado son poco frecuentes, como por no disponer de estimaciones alternativas del valor razonable (IFRS, 2014).

Las pérdidas o ganancias derivadas de un cambio en el valor razonable de las inversiones inmobiliarias se reflejarán en la cuenta de resultados, debiendo la entidad valorar las propiedades a su valor razonable a la fecha que presente información.

Para la determinación del valor razonable de una propiedad de inversión se tomará en cuenta el mercado real existente para ese tipo de propiedad a la fecha de presentación de la información, las expectativas del mercado, los ingresos por alquiler derivados de los arrendamientos, los posibles vendedores y compradores así como las expectativas de los inversionistas (IFRS, 2014). En este sentido, es recomendable pero no es obligatorio que las valoraciones se lleven a cabo por un tasador independiente que tenga una calidad profesional reconocida. 


\section{Modelo de costos}

En caso que la entidad valore sus propiedades de inversión, utilizando el modelo de costos, las propiedades se contabilizarán de acuerdo a la NIC16 (Propiedad, planta y equipo) sin que por ello dejen de clasificarse como propiedades de inversión.

\section{Transferencias}

En lo relativo a las transferencias ya sea de propiedades de inversión a otros activos o de éstos a propiedades inversión, únicamente procederán cuando haya evidencia del cambio del uso de la propiedad, por ejemplo:

\section{Tabla 4}

Transferencias

\begin{tabular}{|l|}
\hline 1.- El inicio de la ocupación por parte del propietario (de propiedades de \\
inversión a propiedad, planta y equipo) \\
\hline $\begin{array}{l}\text { 2.- El inicio de un desarrollo con intención de venta (de propiedades de } \\
\text { inversión a inventarios) }\end{array}$ \\
\hline $\begin{array}{l}\text { 3.- El fin de la ocupación por parte del dueño (de propiedad, planta y } \\
\text { equipo a propiedades de inversión) }\end{array}$ \\
\hline $\begin{array}{l}\text { 4.- El inicio de una operación de arrendamiento operativo (de inventarios a } \\
\text { propiedades de inversión) }\end{array}$ \\
\hline
\end{tabular}

Las propiedades de inversión se darán de baja en el Estado de Situación Financiera en el momento en que sean vendidas o cuando queden permanentemente retiradas de uso y no se espere que generen beneficios económicos futuros (IFRS, 2014). Ahora bien, es importante destacar que la determinación de si una propiedad se debe clasificar como inversión inmobiliaria o bien como Propiedad, Planta y Equipo, requiere en muchas ocasiones del juicio profesional (Guía NIIF, 2014, 96).

\section{NORMATIVIDAD FINANCIERA MEXICANA}

\section{Aspectos generales}

Las normas de información financieras mexicanas no contemplan hasta el momento el concepto de propiedades de inversión, sin embargo, a través de su circular número 55 dicta los lineamientos sobre aplicación supletoria de la NIC 40, así mismo en caso de que las entidades no optarán por aplicar la citada circular no.55, tendrían que manejar sus inversiones inmobiliarias por medio de la NIF C-6 relativa a propiedad, planta y equipo. A continuación, vamos a realizar un análisis de los elementos más significativo de la Circular 55 y la NIF C-6, que afectan a nuestro estudio: 


\section{Circular 55}

En lo referente a la circular 55, la Comisión de Principios de Contabilidad determinó que la NIC 40 representa una particularidad dentro del entorno mexicano, por lo tanto, estableció que se tiene que aplicar supletoriamente la NIC 40 únicamente en lo referente al Modelo del Costo, para ello deberán las entidades: 1.- Reclasificar las propiedades de inversión, presentándola como tales en el Estado de Situación Financiera, dentro del activo no corriente.

2.- Continuar con la valuación por el método de costo (actualizado en los términos del boletín B-10), como si estuvieran aún dentro de inmuebles, maquinaria y equipo; $\mathrm{y}$

3.- Hacer las revelaciones aplicables al modelo de costo requeridas por la NIC40.

Cabe destacar que el marco conceptual mexicano está estructurado en base al modelo del costo histórico es por ello de la pertinencia de aplicar el modelo de costo en lugar del modelo de valor razonable.

\section{$\underline{\text { NIF C-6 }}$}

La propiedad, planta y equipo son bienes tangibles que tienen por objeto: 1.- El uso o usufructo de los mismos en beneficio de la entidad

2.- La producción de artículos para su venta; y

3.- La prestación de servicios por la entidad a su clientela o al público en general.

Al no existir una norma particular sobre inversiones inmobiliarias en México estas quedarían inmersas dentro de la NIF C-6 relativa a propiedad, planta y equipo, ya que son bienes tangibles que se usan para generar beneficios a la entidad.

A continuación se hace un análisis de los principales puntos de la NIF C-6, referente al objetivo, alcance, normas de valuación y normas de presentación.

\section{Objetivo y alcance}

La NIF C-6 tiene como objetivo establecer la normatividad relativa a la valuación, presentación y revelación de propiedad, planta y equipo conocidos comúnmente como activos fijos. Esta norma debe de aplicarse a todos los activos clasificados como propiedad, planta y equipo de las entidades que emitan estados financieros en los términos de la NIF A-3 (Necesidades de usuarios y objetivos de los estados financieros), esta NIF C-6 no se aplicará a activos fijos clasificados como mantenidos para la venta, activos biológicos, activos para exploración de recursos minerales así como aquellas inversiones en los fundos mineros. (CINIF, 2015)

\section{Reconocimiento inicial}

Al igual que en la normatividad internacional, para que un componente de propiedad, planta y equipo (PPyE en adelante) pueda reconocerse tanto 
inicialmente como posteriormente debe generar posibles beneficios económicos que fluyan a la entidad así como que su costo de adquisición pueda valuarse confiablemente.

El costo de adquisición de un componente de PPyE comprenderá el precio de adquisición, los impuestos y gastos de importación, impuestos indirectos no recuperables, los honorarios y cualquier otro gasto que recaiga sobre la adquisición, incluyendo los costos necesarios para dejar el lugar donde se ubicara el activo completamente funcional y la estimación inicial de costos relacionados con la desinstalación del activo cuando esta sea obligatoria. (CINIF, 2015)

En lo relativo a terrenos y edificios, la NIF C-6 señala que el costo de adquisición de un terreno incluirá el precio de adquisición, los honorarios y gastos notariales, las indemnizaciones pagadas sobre la propiedad a terceros, las comisiones a agentes, los impuestos de traslación de dominio, los honorarios de abogados y los costos de urbanización para su uso. En cuanto a los edificios su costo inicial de adquisición incluirá la construcción, instalaciones, equipo de carácter permanente, permiso de construcción, honorarios de arquitectos e ingenieros entre otros relacionados con la edificación del inmueble.

\section{Reconocimiento posterior}

La NIF C-6 no reconoce el método del valor razonable, por lo tanto para reconocimientos posteriores al inicial las entidades determinarán el valor de los componentes de PPyE de la siguiente forma:

Tabla 5

Método de Costo

\begin{tabular}{|c|}
\hline Costo de Adquisición \\
\hline (menos) Depreciación Acumulada \\
\hline (menos) Pérdidas por deterioro Acumuladas \\
\hline (igual) Valor en libros \\
\hline
\end{tabular}

\section{Depreciación}

La NIF C-6 define a la depreciación como la distribución sistemática y razonable en resultados del monto depreciable de un activo fijo a lo largo de su vida útil, entre las consideraciones más importantes que maneja la norma sobre la depreciación se enumeran las siguientes:

1. Cada componente con un costo de adquisición importante con relación al costo total de adquisición de un elemento de PPyE debe depreciarse de forma separada. 
2. El monto a depreciar se determina dividiendo el resultado de restar al monto de adquisición el valor residual entre la vida útil.

3. El periodo de depreciación es aquel que abarca desde la fecha en que un componente de PPyE esté listo para usarse hasta la fecha en que dicho componente sea clasificado como mantenido para la venta y se produzca su baja.

4. La vida útil de un componente se define con base en la utilidad que se espera que aporte a la entidad y para su determinación influyen factores como el desgaste físico, la obsolescencia técnica, límites legales y la utilización prevista del componente.

5. Entre los métodos de depreciación que señala la norma encontramos el método de actividad, el de línea recta, el de cargos decrecientes y el de depreciación especial.

\section{Depreciación de terrenos y edificios}

La citada norma establece que los terrenos y edificios deben de reconocerse por separado incluso si han sido adquiridos de forma conjunta, debido a que los terrenos tienen una vida ilimitada y no están propensos a la depreciación, mientras los edificios tienen una vida limitada y por lo tanto son propensos a la depreciación, el incremento en el valor de un terreno donde está instalado un edificio no afecta el monto depreciable de dicho edificio. En relación con el deterioro de un componente, una entidad deberá aplicar el Boletín C15, para la determinación del mismo.

\section{Normas de presentación y revelación}

Las PPyE deben presentarse en el activo no circulante dentro del Estado de Posición Financiera, deduciendo de su costo tanto la depreciación acumulada como las pérdidas por deterioro y debe clasificarse en componente no sujetos a la depreciación como terrenos, activos en construcción por mencionar algunos y componente sujetos a depreciación como edificios, maquinaria, etcétera. La entidad revelará entre otras cosas información sobre las bases de reconocimiento inicial, los métodos de depreciación utilizados, vidas útiles, tasas de depreciación utilizadas, costo de adquisición, depreciación y deterioro acumulado al inicio y al final de cada periodo

\section{DIFERENCIAS ENTRE LA NIC 40 Y LA NIF C-6}

Partiendo del análisis de los principales puntos de las normas, en concreto, la NIC 40 de las IFRS y la NIF C-6 de las normas mexicanas en relación con las inversiones inmobiliarias (edificios y terrenos), se puede determinar que la diferencia más significativa radica en el uso del modelo del valor razonable por parte de la NIC 40, para valuaciones posteriores a la inicial tanto para edificios como para terrenos. Por su parte, las NIF mexicanas, en primer lugar carecen de 
una norma específica que trate dichas inversiones inmobiliarias, razón por la cual estas se tendrán que clasificar como componentes de PPyE, sometiendo a los edificios a un proceso de depreciación que les generará una pérdida de valor sistemático año con año a diferencia de lo que ocurriría con la utilización de la NIC 40, donde estos edificios aumentarán su plusvalía o la disminuirían periodo tras periodo con base en las condiciones del mercado inmobiliario, trayendo como consecuencia la emisión de unos estados financieros más fiables. En lo que respecta a los terrenos la NIF C-6 no contempla la depreciación de los mismos pero a su vez no maneja la posibilidad de una revaluación, por lo tanto permanecerían estáticos dentro del estado de situación financiera año con año, situación que, en nuestra opinión, no es muy realista, caso contrario sucedería al aplicar la NIC 40, ya que al igual que en los edificios se determinaría el valor razonable de los terrenos cada vez que se fuera a presentar información financiera.

Las NIF mexicanas contienen la circular No.55 en la que se establece la posibilidad de utilizar supletoriamente el método de costos de la NIC 40 para la valoración de edificios y terrenos. En términos generales, la normatividad mexicana permite la utilización supletoria de las IFRS en aquellos casos donde no exista una norma específica siempre y cuando no se interpongan al marco conceptual mexicano, dicho marco está estructurado basándose en el costo histórico, razón por la cual no permite la utilización del modelo del valor razonable de la NIC 40, situación que nos remite a la relación NIC40-NIF C6, donde los edificios perderán sistemáticamente su valor por el uso de la depreciación.

Ante esta situación, aquellas empresas mexicanas cuyo activo no corriente esté basado principalmente en edificios y terrenos o aquellas que tengan una importante inversión en los mismos, se verán afectados significativamente en su información financiera con el uso de la normatividad mexicana, ya que año tras año registrarán una pérdida de valor de sus edificios en el estado de situación financiera y a su vez no verán reflejado en la cuenta de resultados las posibles ganancias derivadas del aumento de plusvalía de los inmuebles. Es por ello, que la adopción de las IFRS en lugar de las NIF mexicanas surge como una opción para estas empresas.

En el siguiente apartado analizamos el impacto que tendría el uso de las NIC en los Estados Financieros en México, para ello, vamos a presentar un extracto de los Estados Financieros de una entidad mexicana, centrándonos en los Estados de Situación Financiera y los Estados de Resultados y otros resultados integrales.

\section{IMPACTO DEL USO DE LA NIC 40 EN LOS ESTADOS FINANCIERO}

El análisis de las diferencias arroja que el punto central de estas radica en el aumento de valor de las inversiones inmobiliarias derivada de la ganancia por valuación y que tendría un impacto en el resultado de la entidad, es por ello que a continuación se hace un análisis del activo no corriente y su impacto en el resultado. 
El uso de la normatividad financiera internacional, en especial el de NIC 40, tendría un impacto positivo en las finanzas de la empresa como lo demuestra el Balance y el Estado de Resultados emitidos con base en IFRS así como los ratios económicos.

Tabla 6

Estado de Situación Financiera Comparativo COMPARATIVO IFRS - NIF

Estados de situación financiera al 31 de diciembre de 2014 (en miles de pesos)

\begin{tabular}{|c|c|c|c|c|c|}
\hline Activo & IFRS & $\%$ & NIF & $\%$ & Var \\
\hline \multicolumn{6}{|l|}{ Activo circulante: } \\
\hline Efectivo y equivalentes de efectivo & $\$ 1,211$ & 0.15 & $\$ 1,211$ & 0.17 & -0.01 \\
\hline Cuentas por cobrar a partes relacionadas & 109,730 & 13.99 & 109,730 & 15.18 & -1.18 \\
\hline Otras cuentas por cobrar & 332 & 0.04 & 332 & 0.05 & 0.00 \\
\hline Total del activo corriente & 111,273 & 14.19 & 111,273 & 15.39 & -1.20 \\
\hline Propiedades de inversión & 672,778 & 85.80 & 611,589 & 84.60 & 1.20 \\
\hline Mobiliario y equipo, neto & 30 & 0.00 & 30 & 0.00 & 0.00 \\
\hline Total del activo no corriente & 672,808 & 85.81 & 611,619 & 84.61 & 1.20 \\
\hline Total Activo & $\$ 784,081$ & 100.00 & $\$ 722,892$ & 100.00 & 0.00 \\
\hline \multicolumn{6}{|l|}{ Pasivo } \\
\hline \multicolumn{6}{|l|}{ Pasivo circulante: } \\
\hline Impuestos por pagar y gastos acumulados & $\$ 1,689$ & 0.22 & $\$ 1,689$ & 0.23 & -0.02 \\
\hline Cuentas por pagar a partes relacionadas & 343 & 0.04 & 343 & 0.05 & 0.00 \\
\hline Total pasivo corriente & 2,032 & 0.26 & 2,032 & 0.28 & -0.02 \\
\hline Impuestos a la utilidad diferidos & 159,424 & 20.33 & 141,068 & 19.51 & 0.82 \\
\hline Total del pasivo & 161,456 & 20.59 & 143,100 & 19.80 & 0.80 \\
\hline \multicolumn{6}{|l|}{ Patrimonio : } \\
\hline Capital social & 31,260 & 3.99 & 31,260 & 4.32 & -0.34 \\
\hline Utilidades retenidas & 591,365 & 75.42 & 548,532 & 75.88 & -0.46 \\
\hline Total patrimonio & 622,625 & 79.41 & 579,792 & 80.20 & -0.80 \\
\hline Total Patrimonio + Pasivo & $\$ 784,081$ & 100.00 & $\$ 722,892$ & 100.00 & 0.00 \\
\hline
\end{tabular}


Tabla 7

Estado de Resultado Integral Comparativo

COMPARATIVO IFRS-NIF

Estados de resultados y otros resultados integrales al 31 de diciembre de 2014

(En miles de pesos)

\begin{tabular}{|l|c|c|c|c|c|}
\hline & IFRS & $\mathbf{\%}$ & NIF & $\%$ & Índice \\
\hline Operaciones Continuas & $\$ 73,342$ & 100.00 & $\$ 46,545$ & 100.00 & 157.57 \\
\hline Ingresos por arrendamiento & $\$ 41,863$ & 57.08 & $\$ 41,863$ & 89.94 & 100.00 \\
\hline Costo por arrendamiento & $-1,606$ & -2.19 & $-1,606$ & -3.45 & 100.00 \\
\hline Utilidad Bruta & 40,257 & 54.89 & 40,257 & 86.49 & 100.00 \\
\hline & & & & & \\
\hline Gastos de operación & $-2,924$ & -3.99 & $-2,924$ & -6.28 & 100.00 \\
\hline & & & & & \\
\hline Ganancia en valuación de propiedades de inversión & 26,797 & 36.54 & 0 & 0.00 & 0.00 \\
\hline Depreciación & 0 & 0.00 & $-3,969$ & -8.53 & 0.00 \\
\hline BAII(BAIT) & 64,130 & 87.44 & 33,364 & 71.68 & 192.21 \\
\hline & & & & & \\
\hline Ingreso por intereses & 4,682 & 6.38 & 4,682 & 10.06 & 100.00 \\
\hline & & & & & \\
\hline Utilidad antes de impuestos a la utilidad \\
(BAI/BAT)
\end{tabular}

\section{Impacto en el activo no corriente}

El principal rubro que impacta la NIC 40 en el caso que se muestra, son las propiedades de inversión que, para 2014 aumentaron su valor derivado de la ganancia por valuación, pasando de $\$ 642,192$ en 2013 a $\$ 672,778$ para el año en mención, lo que significa un aumento del $4 \%$ en el valor de los bienes inmuebles de la entidad, caso contrario sucede con la aplicación de la normatividad mexicana que al estar afecta a la depreciación según lo establecido en la NIF C-6, sufren una disminución en su valor pasando de $\$ 611,769$ en 2013 a un valor en libros de $\$ 607,800$ para 2014 , una pérdida de valor del $0.65 \%$. 


\section{Impacto en el beneficio}

El aumento en el valor de las propiedades de inversión en el caso que hemos expuesto, derivado de la ganancia por valuación de inmuebles tiene un impacto directo en la determinación del resultado del ejercicio así como en los impuestos diferidos. En este orden de ideas tenemos que el pasivo diferido tuvo un incremento del $5 \%$ con relación a 2013 , pasando de $\$ 151,240$ a $\$ 159,424$ para 2014, lo que trae como consecuencia que la totalidad de la deuda sufra un incremento por el mismo porcentaje y que el impuesto a la utilidad se incremente en un 30\%, pasando de $\$ 12,359$ en 2013 a $\$ 17,823$ en 2014, a pesar del incremento en los impuestos diferidos por el uso de las IFRS y de que en los estados financieros bajo NIF Mexicanas presentan un pasivo diferido menor $(\$ 141,068)$ y por ende un menor impuesto a la utilidad (\$7,910 para 2014), la utilización de la NIC 40 permite a la entidad incrementar su utilidad después de impuestos en un $40 \%$ con relación a la utilidad neta obtenida con base en NIF Mexicanas.

\section{Tabla 8}

Beneficios después de Impuestos según IFRS y NIF Mexicanas

\begin{tabular}{|c|c|c|c|}
\hline BDI IFRS & $\begin{array}{c}\text { BDI NIF } \\
\text { Mexicanas }\end{array}$ & Diferencia & Variación \\
\hline $\mathbf{\$ 5 0 , 9 8 9}$ & $\$ 30,136$ & $\$ 20,853$ & $40.89 \%$ \\
\hline
\end{tabular}

El rubro de las utilidades es clave, ya que si se aplicara la NIC 40 tendría lugar un impacto de manera más considerable en forma indirecta, como lo demuestra la tabla anterior, pero no únicamente la utilidad neta se ve impactada sino también la utilidad operativa, la utilidad antes de impuestos y la ganancia por acción como lo demuestra los siguientes ratios de utilidad: 
Tabla 9

Comparativos Ratios Económicos

\begin{tabular}{|c|c|c|c|c|c|c|}
\hline \multirow{2}{*}{\multicolumn{2}{|c|}{$\begin{array}{c}\text { COMPARATIVO RATIOS } \\
\text { ECONÓMICOS } \\
2014\end{array}$}} & \multirow{2}{*}{\multicolumn{2}{|c|}{ IFRS }} & \multirow{2}{*}{\multicolumn{2}{|c|}{ NIF }} & \multirow[t]{2}{*}{ Var } \\
\hline & & & & & & \\
\hline \multirow{2}{*}{$\begin{array}{l}\text { Margen de utilidad } \\
\text { bruta }\end{array}$} & Utilidad bruta & 40,257 & $96 \%$ & 40,257 & $96 \%$ & $0 \%$ \\
\hline & Ventas & 41,863 & & 41,863 & & \\
\hline \multirow{2}{*}{$\begin{array}{l}\text { Margen de utilidad } \\
\text { operativa }\end{array}$} & BAII & 64,130 & $153 \%$ & 33,364 & $80 \%$ & $73 \%$ \\
\hline & Ventas & 41,863 & & 41,863 & & \\
\hline \multirow{2}{*}{$\begin{array}{l}\text { Margen de utilidad } \\
\text { antes de impuestos }\end{array}$} & BAI & 68,812 & $164 \%$ & 38,046 & $91 \%$ & $73 \%$ \\
\hline & Ventas & 41,863 & & 41,863 & & \\
\hline \multirow{2}{*}{$\begin{array}{l}\text { Margen de utilidad } \\
\text { neta }\end{array}$} & BDI & 50,989 & $122 \%$ & 30,136 & $72 \%$ & $50 \%$ \\
\hline & Ventas & 41,863 & & 41,863 & & \\
\hline \multirow[t]{2}{*}{$\begin{array}{l}\text { Ganancia por } \\
\text { acción }\end{array}$} & BDI & 50,989 & $8 \%$ & 30,136 & $5 \%$ & $3 \%$ \\
\hline & No. Acciones & 625,709 & & 625,709 & & \\
\hline \multirow{2}{*}{$\begin{array}{l}\text { Tasa de } \\
\text { participación } \\
\text { de gastos }\end{array}$} & Gastos & 4,530 & $11 \%$ & 8,499 & $20 \%$ & $-9 \%$ \\
\hline & Ventas & 41,863 & & 41,863 & & \\
\hline
\end{tabular}

Se observa que la ganancia en valuación de propiedades de inversión derivado del uso de la NIC 40, produce márgenes de utilidad arriba del 100\% para BAII, BAI y BDI, lo que significa que la totalidad de las ventas son utilidades para los socios y que impactará positivamente en el incremento del patrimonio de la entidad 
como lo demuestra el Estado Situación Financiera presentado con base en normas internacionales.

Por lo tanto el uso de la NIC 40 no impacta directamente en los ingresos de la entidad pero sí en sus márgenes de utilidad y en su patrimonio, que si se compara con los resultados que arrojan los estados financieros con base en normas financieras mexicanas es notaria la diferencia porque mientras que las NIF Mexicanas tienen un margen de utilidad para los socios del $72 \%$ de la totalidad de las ventas, las normas internacionales les otorgan el $100 \%$ de utilidad sobre las ventas más un plus de $22 \%$.

\section{Gráfico 1}

Comparativo de los rubros afectados dentro del balance por el uso de la NIC-40.

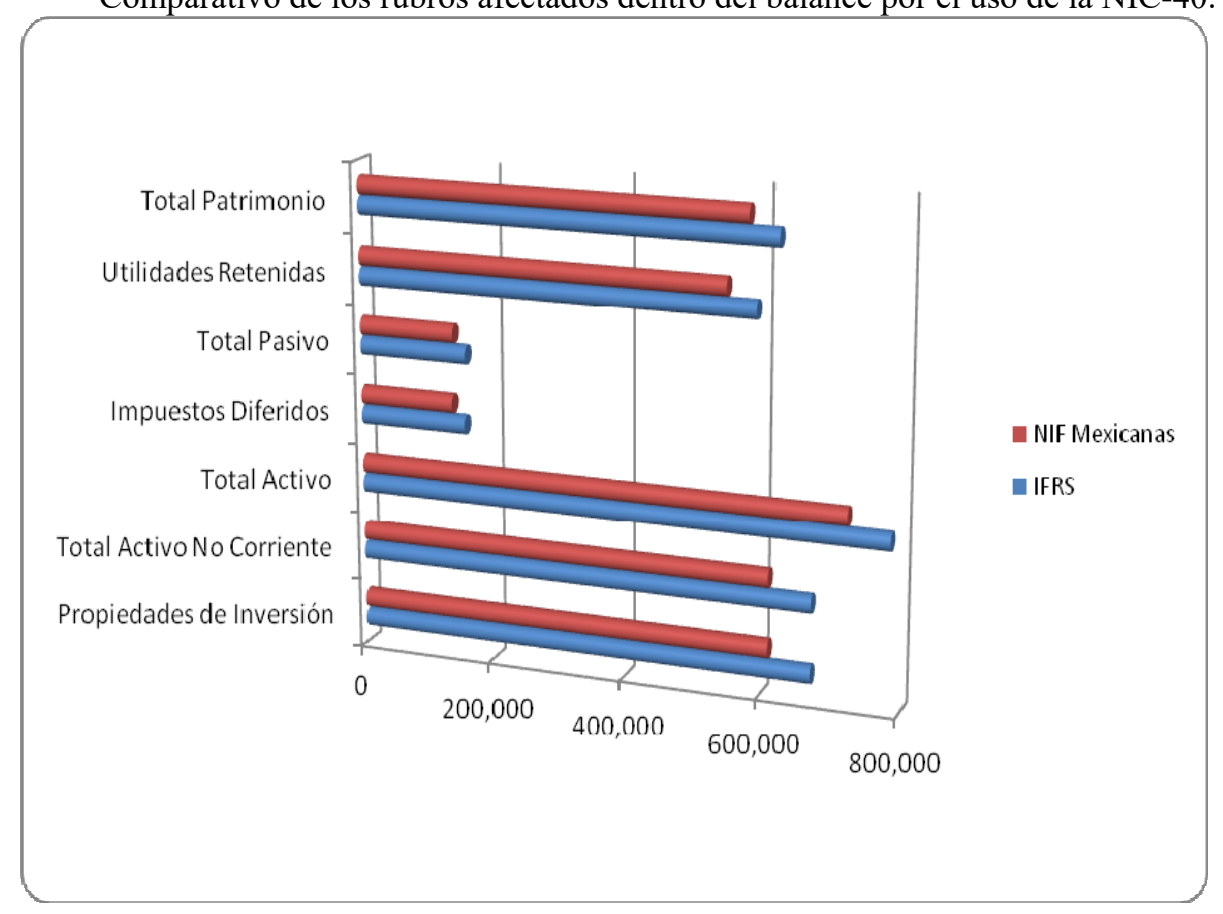




\section{Gráfico 2}

Comparativo de los rubros afectados dentro del resultado por el uso de la NIC-40.

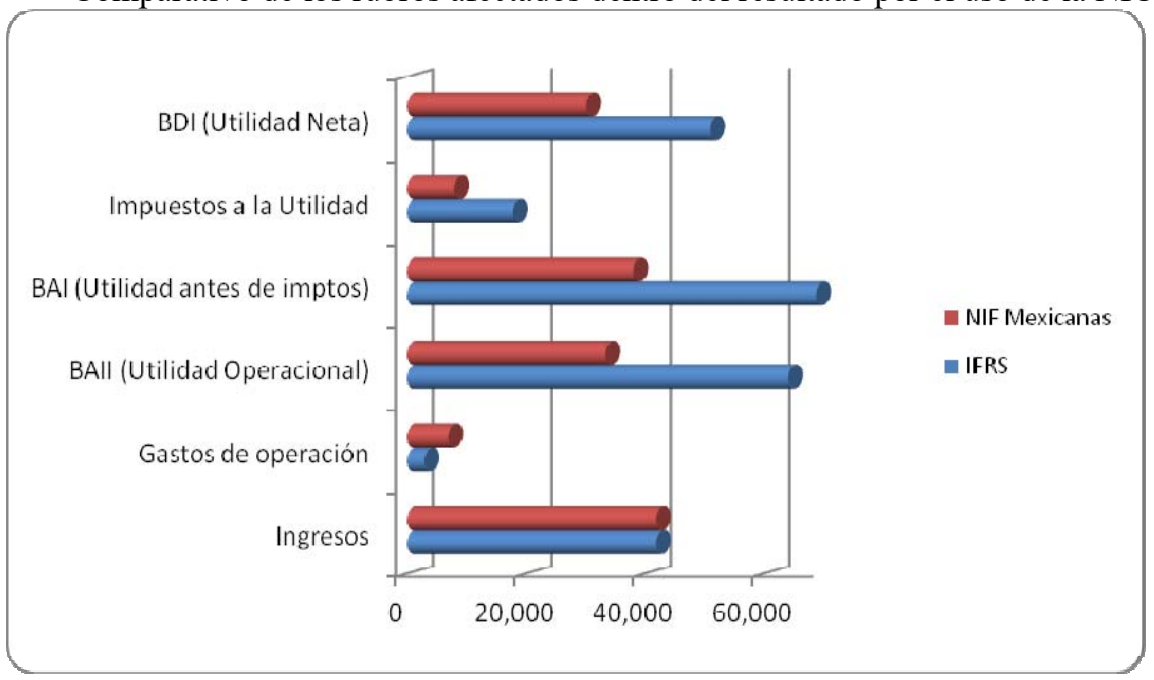

\section{Gráfico 3}

Comparativo IFRS-NIF Mexicanas de los Márgenes de Utilidad

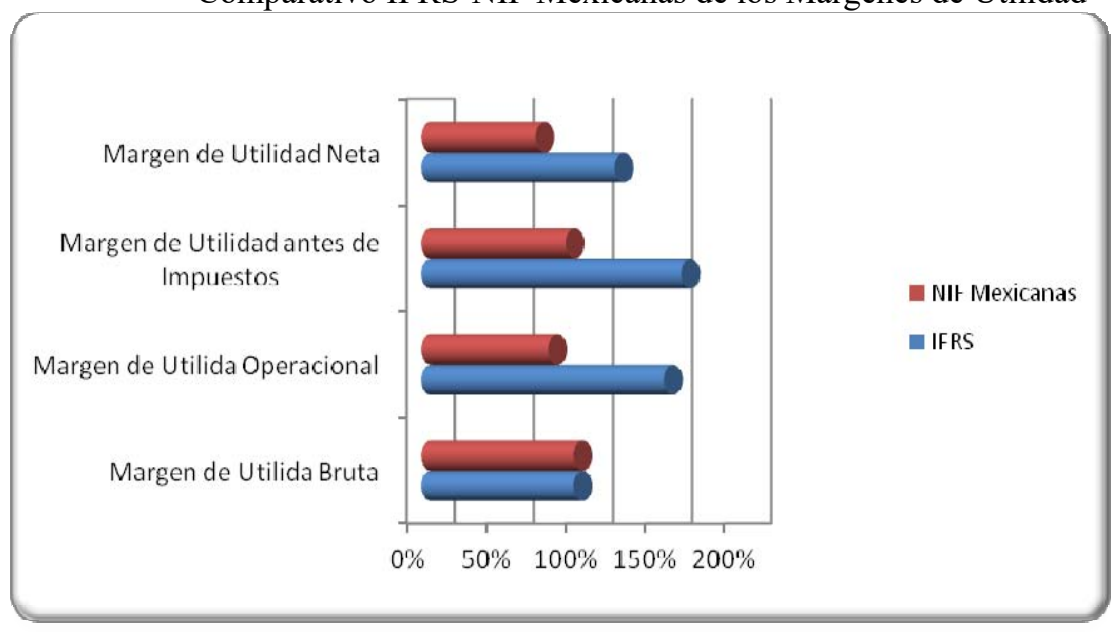

\section{IMPACTO IMPOSITIVO POR EL USO DE LA NIC 40}

La ganancia por valuación en propiedades de inversión derivado del uso de la NIC 40, impacta en los impuestos a las ganancias como se ha mencionado 
anteriormente, a continuación se analiza el impacto que tiene el uso de esta norma tanto en el impuesto corriente como en el diferido.

\section{Impuesto corriente}

Para la Ley del Impuesto sobre la Renta (LISR) que es la norma legal mexicana para la determinación del impuesto a la utilidad, no se considera como un ingreso acumulable en términos fiscales la ganancia por valuación de propiedades de inversión según lo establecido en artículo 16 de la mencionada ley y que a la letra dice: "Para los efectos de este Título, no se consideran ingresos los que obtenga el contribuyente por aumento de capital, por pago de la pérdida por sus accionistas, por primas obtenidas por la colocación de acciones que emita la propia sociedad o por utilizar para valuar sus acciones el método de participación ni los que obtengan con motivo de la revaluación de sus activos y de su capital." (Art16LISR) (LISR, 2014)

Basándonos en el párrafo anterior, el uso de la NIC 40 en lo referente a la ganancia por valuación, no tiene un impacto fiscal para la determinación del impuesto corriente de la entidad.

En lo referente a la depreciación esta solo muestra diferencia desde un punto de vista contable ya que el uso de la normatividad financiera exige que las inversiones inmobiliarias sean depreciadas (NIF C-6) mientras que la normatividad internacional con base en la NIC 40 irá revaluando su valor al final del ejercicio por el método del valor razonable, sin embargo esta diferencia entre normatividades no tiene un impacto fiscal ya que para LISR, las inversiones inmobiliarias se consideran inversiones según lo establecido en el art.32 " Para los efectos de esta Ley, se consideran inversiones los activos fijos, los gastos y cargos diferidos y las erogaciones realizadas en periodos pre operativos, de conformidad con los siguientes conceptos: Activo fijo es el conjunto de bienes tangibles que utilicen los contribuyentes para la realización de sus actividades y que se demeriten por el uso en el servicio del contribuyente y por el transcurso del tiempo." (LISR, 2014).

Por lo tanto las inversiones inmobiliarias se consideraran según lo establecido en LISR como un activo fijo y estarán afectas a una depreciación fiscal y a las demás reglas establecidas para las inversiones de acuerdo a la Sección II; Capítulo II; Titulo II (art.31 al 38) de LISR. En consecuencia la depreciación fiscal será exactamente la misma utilizando una u otra normatividad ( $\$ 5,809$ para 2014) ya que se determina de acuerdo a la ley y no a las normas financieras.

A continuación, presentamos el caso de la empresa antes analizada centrándonos en el análisis comparativo del Impuesto Anual a las Ganancias 


\section{Tabla 10}

Comparativo Impuesto Anual a las Ganancias

(Conciliación: Contable-Fiscal)

\begin{tabular}{|c|c|c|c|c|}
\hline Origen & Concepto & NIF's Mexicanas & IFRS & Diferencia \\
\hline & Utilidad o pérdida neta (Contable) & 20,223 & 50,989 & $-30,766$ \\
\hline más & Ingresos fiscales no contables & - & - & \\
\hline \multirow[t]{5}{*}{ más } & Deducciones contables no fiscales & 21,804 & 17,835 & 3,969 \\
\hline & Depreciación Contable & 3,969 & - & \\
\hline & $\begin{array}{l}\text { Gastos que no reúnen requisitos } \\
\text { fiscales }\end{array}$ & 11 & 11 & \\
\hline & ISR del ejercicio & 9,639 & 9,639 & \\
\hline & ISR Diferido & 8,185 & 8,185 & \\
\hline \multirow[t]{4}{*}{ menos } & Deducciones fiscales no contables & 9,897 & 9,897 & 0 \\
\hline & Ajuste anual por inflación deducible & 3,954 & 3,954 & \\
\hline & Depreciación Fiscal & 5,809 & 5,809 & \\
\hline & Estimaciones & 133 & 133 & \\
\hline \multirow[t]{2}{*}{ menos } & Ingresos contables no fiscales & - & 26,797 & $-26,797$ \\
\hline & Otros ingresos contables no fiscales & - & 26,797 & \\
\hline \multirow[t]{8}{*}{ igual } & $\begin{array}{l}\text { Utilidad o pérdida fiscal antes de } \\
\text { PTU }\end{array}$ & 32,130 & 32,130 & -0 \\
\hline & tasa $30 \%$ & $30 \%$ & $30 \%$ & \\
\hline & & & & \\
\hline & Impuesto Causado & 9,639 & 9,639 & \\
\hline & Pagos Provisionales & & & \\
\hline & SAT & 9,848 & 9,848 & \\
\hline & Retenciones & 74 & 74 & \\
\hline & Impuesto a cargo (a favor) & (284) & (284) & \\
\hline
\end{tabular}

El cuadro anterior sobre la conciliación contable-fiscal, muestra que las diferencias entre el uso de las normatividad internacional o la mexicana son de carácter contable y no tienen impacto en la determinación del impuesto corriente, que para 2014 fue de 9,639. 


\section{Impuesto diferido}

A diferencia de los impuestos corrientes donde el uso de la NIC 40 tiene un impacto nulo, en los impuestos diferidos el uso de la citada norma impacta directamente en la base para la determinación de los mismos como lo refleja la siguiente tabla.

Tabla 11

Determinación del Impuesto Diferido

\begin{tabular}{|l|c|c|c|}
\hline \multicolumn{1}{|c|}{ Concepto } & IFRS & NIF Mexicanas & Diferencia \\
\hline Propiedades de Inversión (fiscal) & 0 & 0 & 0 \\
\hline $\begin{array}{l}\text { Propiedades de Inversión } \\
\text { (contable) }\end{array}$ & 672,779 & 611,589 & 61,190 \\
\hline Diferencia (Contable-Fiscal) & $(672,779)$ & & $(61,190)$ \\
\hline Tasa Fiscal & $30 \%$ & $(611,589)$ & \\
\hline Impuesto Pasivo diferido & $(201,834)$ & $30 \%$ & $(18,357)$ \\
\hline (-)Impuesto de Activos & 42,409 & $(183,477)$ & 0 \\
\hline (=) Pasivo Diferido 2014 & $(159,424)$ & 42,409 & $(18,357)$ \\
\hline (-) Pasivo diferido 2013 & $(151,240)$ & $(141,067)$ & $(8,443)$ \\
\hline (=) Impuesto Diferido 2014 & $(8,184)$ & 1,729 & $(9,913)$ \\
\hline (+) Impuesto Corriente & $(9,639)$ & $(9,639)$ & $(7,910)$ \\
\hline (=) Impuesto a la Utilidad & $(17,823)$ & & \\
\hline
\end{tabular}

El uso de la NIC 40 incrementa el impuesto diferido como lo demuestra la tabla 9, pasando de un diferido a cargo según IFRS por $\$ 8,184$ a un diferido a favor de $\$ 1,729$ por la aplicación de la normatividad mexicana. Por lo tanto el uso de la normatividad internacional en lugar de las normas mexicanas impacta en el impuesto a la utilidad únicamente en lo concerniente a los impuestos diferidos no así en el impuesto corriente.

\section{CONCLUSIONES}

Teniendo en cuenta los estados financieros emitidos de una empresa que realiza sus actividades empresariales en México, se ha partido de la base de la aplicación en sus estados financieros tomando como base las normas financieras mexicanas. Posteriormente, se ha realizado una simulación de los mismos estados financieros tomando como hipótesis de aplicación de las normas financieras internacionales y hemos llegado a las siguientes conclusiones en relación a la posible aplicación de la NIC 40 respecto a las inversiones inmobiliarias como alternativa a las normas locales en México: 
Le proporciona a la entidad un enfoque más real sobre el valor de sus bienes inmuebles y por lo tanto sus estados financieros tienen una imagen más fiel de su situación financiera, esto es derivado por el uso del modelo del valor razonable en lugar del modelo de costo, lo cual incrementa el activo no corriente así como los márgenes de utilidad de la entidad como consecuencia de la ganancia por valuación, lo que impacta directamente en el patrimonio de la entidad, el cual registra un incremento, y es precisamente en este rubro donde el uso del valor razonable reflejaría más fielmente la situación financiera de la empresa ya que al valuar anualmente los inmuebles estos reflejarán su costo real en el mercado.

En relación con los impuestos el uso de la NIC 40 solo tiene impacto en los impuestos diferidos no así en los corrientes los cuales son exactamente los mismos utilizando la normatividad internacional o mexicana ya que su determinación se basa en leyes no en normas, en relación con los impuestos diferidos su incremento no representa un impacto financiero importante para la entidad.

La diferencia más importante en relación con las NIF Mexicanas radica en el uso del modelo del valor razonable para el reconocimiento posterior del valor de las inversiones inmobiliarias, lo cual mejora la situación financiera de la empresa así como su rendimiento, siendo además una información fundamental a tener en cuenta en la toma de decisiones de usuarios tanto internos como externos de la entidad.

Para concluir, proponemos la aplicación de las Normas Internacionales de Información Financiera y en concreto, la NIC 40, ya que es el objeto del estudio de este trabajo, puesto que, el uso de las normas de información financiera internacionales tiene un impacto financiero positivo en la entidad y representa una mejor opción que las normas de información financiera mexicanas en la empresa en cuestión.

\section{BIBLIOGRAFÍA}

APÓSTOL, S. y DAZA, D. (2014). "Las propiedades de inversión y el valor razonable". Disponible en: http:/www.fccpv.org/cont3/data/files/ Boletin_05_FCCPV.pdf/ (accedido el 29 septiembre, 2015).

ASESOR EMPRESARIAL (2014). NIC 4. Inversiones Inmobiliarias. Revista de asesoría especializada. Lima, Perú.

AZUA, D. y SILVA, B. (2006). "Alcances sobre el concepto de valor razonable". Disponible en: http://www.capic.cl/capic/media/ART5SilvaAzua.pdf/ (accedido el 29 septiembre, 2015).

BARRIENTOS, M. (2013). "Las propiedades de inversión y el valor razonable en el sector inmobiliario". Disponible en: http://www.bdigital.unal.edu.co/ 11383/1/mariasoniabarrientosestrada.2013.pdf/ (accedido el 30 septiembre, 2015). 
COLEGIO DE CONTADORES PÚBLICOS DE MÉXICO, CCPM. (2009). "Como determinar el valor razonable". Disponible en: http://www.ccpm.org.mx/veritas/abril2010/determinar_valor_razonable.pdf/ (accedido el 30 septiembre, 2015).

COLLINGS, S. (2012),"Accounting for Assets", Wiley, J.(Ed.), IFRS for dummies. Jhon Wiley \& Sons Ltd, Glasgow, pp.71-87

CONGRESO GENERAL DE LOS ESTADOS UNIDOS MEXICANOS, LISR. (2015). "Título II.De las Personas Morales", Tax,(Ed.), Ley del Impuesto sobre la Renta. Tax, Puebla, pp.43-99.

CONSEJO MEXICANO PARA LA INVESTIGACIÓN Y DESARROLLO DE LAS NORMAS DE INFORMACIÓN FINANCIERA AC, CINIF. (2011). "Sección C. Disposiciones de las NIF, que coinciden con una alternativa de las IFRS", Del Monte, E, Convergencia de las NIF con las IFRS. CINIF, México D.F., pp. 152-153.

CONSEJO MEXICANO PARA LA INVESTIGACIÓN Y DESARROLLO DE LAS NORMAS DE INFORMACIÓN FINANCIERA AC, CINIF. (2015), Normas de Información Financiera. CINIF: Querétaro.

ESCOBEDO, R. (2013). "Auditando el valor razonable". Revista de Contaduría Pública, Vol. 42, n²91, pp. 30-34.

GIORGANA, A. (2011). "La importancia del mayor y mejor uso en el valor razonable de activos inmobiliarios". Disponible en: http://www.imef.org.mx/Descargascomites/FinanzasCorporativas/abril2011f inanzas.pdf/ (accedido el 29 septiembre, 2015).

GRIFFIN, M. (2009), "Financial Statement Analysis", Kaplan, P.(Ed.), MBA Fundamentals Accounting \& Finance. Kalpan Publishing, New York, pp. 163-182.

GUÍA NIIF 2014 para directives, EY Perú.

INTERNATIONAL FINANCIAL REPORTING STANDARD FOUNDATION, IFRS.(2014). "Norma Internacional de Contabilidad 40 Propiedades de Inversión". Disponible en: http://www.ifrs.org/IFRSs/Pages/OfficialUnaccompanied-IFRS-Translations.aspx\#es/ (accedido el 8 octubre, 2015).

INTERNATIONAL FINANCIAL REPORTING STANDARD FOUNDATION, IFRS.(2009). "Módulo 16: Propiedades de Inversión". Disponible en: http://www.ifrs.org/IFRS-for-

SMEs/Documents/Spanish\%20IFRS\%20for\%20SMEs\%20Modules/Update d\%20Spanish\%20IFRS\%20for\%20SMES\%202014/16_Propiedades\%20de \%20Inversion_2013.pdf/ (accedido el 30 septiembre, 2015). 
INTERNATIONAL FINANCIAL REPORTING STANDARD FOUNDATION, IFRS.(2012). "NIIF 13 Medición del valor razonable". Disponible en: http://www.ifrs.org/IFRSs/Documents/IFRS13sp.pdf/ (accedido el 30 septiembre, 2015).

KPMG INTERNATIONAL REPORTING GROUP, KPMG (2012). Las NIIF comentadas. Thomson Reuters Aranzadi: Pamplona.

LITTLE, K. (2012), "Types of Mutual Funds", Buttler-Knight, M.(Ed.), Teach yourself Investing in 24 easy lessons. Alpha, New York, pp.163-168.

RAMIREZ ORELLANA, A. (2007). "NIC 40. Inversiones Inmobiliarias", Partida Doble, $n^{\circ} 185$. Pp. 46-55

RODRÍGUEZ, M., FIDALGO, E., GRANADOS, F., MÉNDEZ, T. y PÉREZ, J. (2009), Análisis financiero y económico: Metodología adaptada al Espacio Europeo de Educación Superior. UCM Editorial Complutense: Madrid.

VALLS, M. (2011),"Valor y principios de valoración", Pirámide, (Ed.), Introducción a las finanzas. Ediciones Piramide, Madrid, pp.246-255. 